\title{
Image analysis for detecting the transverse profile of worn-out rails
}

\author{
M. Guerrieri*, G. Parla**, D. Ticali*** \\ *Faculty of Engineering, University of Perugia, Italy \\ **Faculty of Engineering, University of Palermo, Italy \\ ***Faculty of Engineering, University of Enna "Kore, Italy"
}

\begin{abstract}
Over its useful life a railway track is subject to many mechanical and environmental stresses which gradually lead to its deterioration. Monitoring the wear condition of the railway superstructure is one of the key points to guarantee an adequate safety level of the railway transport system; in this field, the use of high-efficiency laser techniques has become consolidated and implemented in diagnostic trains (e.g. the "Archimede train" and the "Talete train" ) which allow to detect the track geometric parameters (gauge, alignment, longitudinal level, cross level, superelevation defect, etc.) and the state of rail wear (vertical, horizontal, 45-degree etc.) with very high accuracy. The objective of this paper is to describe a new nonconventional procedure for detecting the transverse profile of worn-out rails by means of image-processing technique. This methodological approach is based on the analysis of the information contained in high-resolution photographic images of rails and on specific algorithms which allow to obtain the exact geometric profile and the measurement of the relevant deviations compared to new rails of the same typology.

The analyses and the first results, obtained from laboratory researches, concern rails cross sections taken from railway lines under upgrading. The procedure has shown high precision in the wear evaluation as well as great rapidity in being performed.
\end{abstract}

\section{TRACK IMAgE SEgmentation}

The primary goal of image processing is to make the information content of a given image explicit with regard to the reference application type. All the segmentation methods are defined to process an image through specific algorithms which divide that image into distinct and uniform regions according to a feature set for the segmentation purpose. This operation is the first step to distinguish the region enclosing the object of interest (ROI, region of interest; here the railway track) from the other parts or the background. In general, in order to split a grayscale image into different regions, the starting point is given by two different, but not opposite observations:

- intensity differences between the adjacent pixels are generally recorded in the transit area from a region to another. Therefore, suitable algorithms with first- or second-order derivative filters are available to survey significant discontinuities (edge detection);

- the intensities of pixels belonging to the same region are usually "sufficiently" similar to one another. Pixel similarity in a given region can be established depending on

DOI: 10.5176_2010-2283_2.1.132 their intensity and also on their position.

Obviously, an image partition process must be able to preserve the valuable information along the object contours which in this paper is the starting point for detecting the distances between the rail transverse profiles in different operational conditions, more specifically worn-out and new rails. In light of this, an algorithm has been implemented to solve these problems through a set of functions properly specified. As a matter of fact, after analyzing the peculiarity of the image under consideration, the segmentation procedure detects the image contour or "edge" through Canny's algorithm [1] and other procedures, properly calibrated for the research purpose, which maximize the geometric information achievable from the image edge under examination. In the following steps this procedure solves the complex problem to compare the two track profiles (worn-out and new) and to measure the deviations. The paragraphs below illustrate and formalize the two procedures. In order to analyze the image of a track plane section, the first step of the procedure is the edge detection of the transverse profile through Canny's algorithm. The image edge detection phase is necessary in that digital images are definitely realized by high-resolution equipment but some image pixels could be affected by noise (very rough light scatterings located at the track image boundary), thus making the track edge detection less accurate. More specifically, it is essential to detect the exact position of the image edge even in those parts where it cannot be distinguished, perceptually and/or numerically, from the clear background. Canny's algorithm [1] examines the behavior of the gradient operator applied to a noisy contour.

The quality of the results which can be obtained from Canny's method is accounted for the fact that this method employs two thresholds: one for the detection of the most marked edges, the other for the detection of the roughest edges. The latter are, however, taken into consideration only if they appear to be connected to the marked edges. Therefore, in Canny's algorithm the traditional thresholding approach with a single value needs to be replaced with a double threshold called hysteresis thresholding; the latter is applied after the non-maximum suppression. Notably, set the two thresholds $\mathrm{t} 1$ and $\mathrm{t} 2$ as $\mathrm{t} 1>\mathrm{t} 2$, the algorithm establishes that the pixel located at position $(i, j)$ on the generic chromatic plane $\mathrm{p}^{1}$ of the image $A(I, j, p)$ is a Canny contour if the intensity value of the pixel examined - A(i,j,p) - appears to be superior to the

1 "p" is plane index: $p=1$ Red plane, $p=2$ Green plane, $p=3$ Blue plane 
highest threshold value $\mathrm{t} 1$; whereas the lowest value $\mathrm{t} 2$ denotes the boundary below which the pixel is not certainly a Canny contour. Moreover, the algorithm establishes that in a pixel set the values between $\mathrm{t} 1$ and $\mathrm{t} 2$ are to be estimated as edged only if they appear to be connected to those which are certainly edged, that is those with a value higher than $t 1$. This can be summed up in the following inequalities:

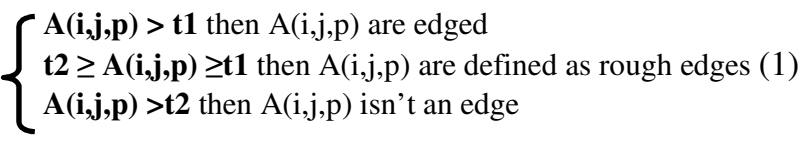

The track edge points obtained by Canny algorithm, denoted with $\{\mathrm{C}\}$, are illustrated in the binary image of Fig. 2, which is an enlargement of a part of the original image (Fig. $1)$.

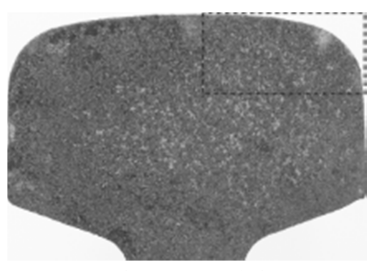

Fig. 1. Track image

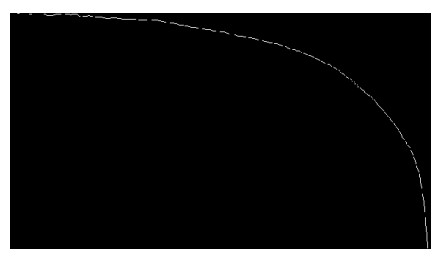

Fig. 2. Binary image (enlargement: red area)

For each contour point $\{\mathrm{C}\}$, by using suitable convolution masks and imposing the congruency on chromatic intensities, it is possible to:

1 calculate the gradient direction;

2 produce two sets $\{\mathrm{A}\}$ (clear) and $\{\mathrm{B}\}$ (dark) for the eight pixels adjacent to each contour point; an example of the pixel allocation procedure to such sets can be seen in the pixel intensities of the image illustrated in Figure 3;3

3 remove any pixels belonging to $\{\mathrm{A}\}$ and $\{\mathrm{B}\}$ at the same time.
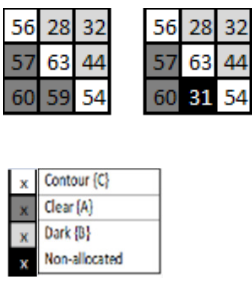

Fig. 3. Allocation to sets $\{A\}$ and $\{B$

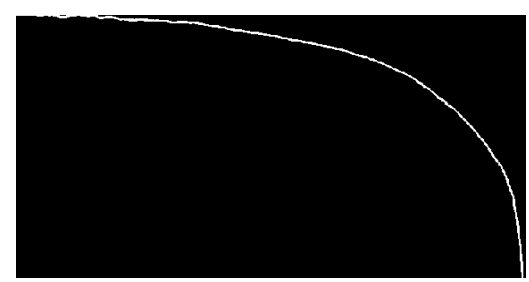

Fig. 4. Binary image without discontinuities

So, a slight edge thickening is obtained, thus closing (by connectivity) and removing any discontinuities (cf. Fig. 4). Moreover, the algorithm is able to distinguish some clearer pixels near the track edge which do not belong to its microwrinkles, as can be seen in Figs. 5,6,7 and 8, obtained from an enlargement of the upper profile of the railhead.

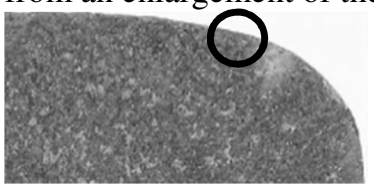

Figure 5 - Part of the original image

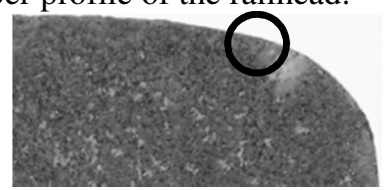

Figure 6 - Part of the filtered original image

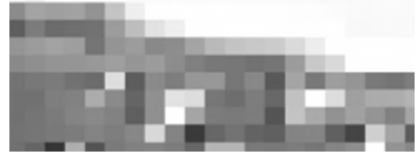

Fig. 7. Enlargement of a part of the original image

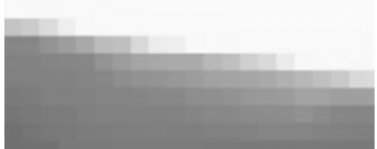

Fig. 8. Enlargement of the filtered original image
Moreover, the procedure further examines the position $(\mathrm{x}, \mathrm{y})$ of every pixel located in the previously detected contour $\{C\}$ and this time modifies the initial information, i.e. that contained in the pixel values along the original image boundary A. This is detailed below.

For every chromatic plane of the analyzed image, a median filter is applied by following this relation:

\section{$\mathbf{M}=\mathbf{A} * \mathbf{B}$}

where template $B$, in this specific case, has a definite dimension [3x3] and a coefficient equal to (1/9).

$$
B=\left(\frac{1}{9}\right) *\left[\begin{array}{lll}
1 & 1 & 1 \\
1 & 1 & 1 \\
1 & 1 & 1
\end{array}\right]
$$

Typically, by a bidimensional signal, like a digital image, a median filter (2) tends to eliminate the noise (high-frequency disturbance), but simultaneously it introduces an image blurring effect to mitigate the "steps" representing the details of the filtered objects (edges, roughness etc.). The implemented procedure iteratively applies the previous filter so as to spread the numerical values of all the pixels which certainly belong to a colorimetrically homogeneous region, located at either the background or track. As a matter of fact, psycho-physiological studies on perception (Wohrer \& Kornprobst, 2009) confirm that edge detection in images and surfaces of separation between object and background is fundamentally based on the difference in color and/or brightness between otherwise homogeneous regions. Therefore the idea is very simple: the image at time $\mathbf{t}$ (iteration step) derives from the initial image convolved with the median filter (2), or:

$$
A(i, j, t)=A_{0}(i, j) x M(i, j, t)
$$

where $(i, j)$ are the pixel position indices within the original matrix A and " $x$ " represents the convolution operator. Obviously, in this spreading process some congruence conditions need to be imposed and therefore it is necessary to correctly attribute every generic pixel at the position $[\mathrm{i}, \mathrm{j}]$ to a colorimetrically homogeneous region (be it the track or background), so as not to eliminate fundamental information when the aim is to study the extent of the track profile lowering, starting from the edge examination. Moreover, since the expanded mean filter (4) tends to remove the information on the edge details, this effect should be mitigated by 
applying another operator which, instead, emphasizes the track edge. In light of the above, downstream from the previous convolution (4), an edge extraction operator called range operator [2] and [3] was applied. This type of (non convolutive) filter allows to replace the central pixel with the range difference of template $\mathrm{B}$ selecting the chromatic intensities of image A, which has a [3x3] size as well. For each deviation $(i, j)$ from template $B$, the value of the central pixel can therefore be formalized as follows:

$$
\begin{aligned}
& R(i, j)=\left[\operatorname { M A X } \left(A_{1-1, j-1} ; A_{i-1, j} ; A_{i-1, j+1} ; A_{i, j-1} ; A_{i, j} ; A_{i, j+1} ;\right.\right. \\
& \left.A_{i+1, j+1}\right)- \\
& \operatorname{MIN}\left(\mathrm{A}_{1-1, j-1} ; \mathrm{A}_{\mathrm{i}-1, \mathrm{j}} ; \mathrm{A}_{\mathrm{i}-1, \mathrm{j}+1} ; \mathrm{A}_{\mathrm{i}, \mathrm{j}-1} ; \mathrm{A}_{\mathrm{i}, \mathrm{j}} ; \mathrm{A}_{\mathrm{i}, \mathrm{j}+1} ;\right. \\
& \left.\left.\mathrm{A}_{\mathrm{i}+1, \mathrm{j}+1}\right)\right]
\end{aligned}
$$

The range values of every [3x3] boundary of all the pixels belonging to the original image A have been sought and highlighted. Such a filter calculates the chromatic intensity values which will fill in a matrix including information on element contours. These values are then subtracted from matrix $M$ which, as previously said, is the result of the convolution expressed by the relation (4). The following equation (6) formalizes the previous difference operation. Therefore, given a generic iteration step $\mathbf{t}$, the suggested algorithm is able to lower the pixel intensities which mark the track plane section contour.

$$
J(i, j, t)=J(i, j)+a b s(M(i, j, t)-R(i, j))
$$

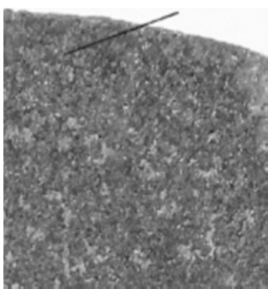

Fig. 9. Reference segment

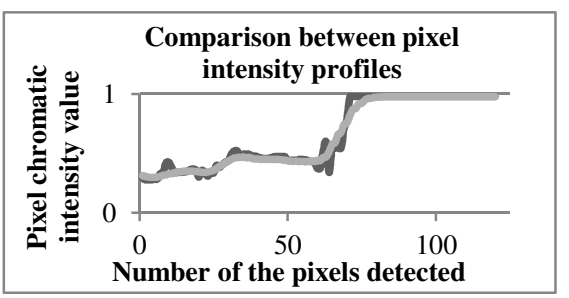

Fig. 10. Chromatic intensity values
Then we detected some uncertainty areas along the point edges where the profile in the eight directions marked a rough variation or, in any case, a skip over a precise threshold level, localized by three constants [Ti, To, Ts]. Such constants are automatically determined by the algorithm in relation to the maximum, minimum and mean deviation values of the two profiles. In these areas, for each iterative step $\mathbf{t}$ the pixel values fill in the matrixes $\{\mathrm{Si}\}$ and $\{\mathrm{Gi}\}$, well distinct from the matrixes $\{\mathrm{S}\}$ and $\{\mathrm{G}\}$ which instead are almost certainly background and track.

$\{\mathrm{S}\}$ : set consisted of pixels which, in the original image $\mathrm{J}$, have such an intensity Ip that $0 \leq \mathrm{Ip} \leq \mathrm{Ti}$; almost certainly, these pixels correspond to background points;

- $\{\mathrm{Si}\}$ : set consisted of pixels which have such an intensity Ip that $\mathrm{Ti}<\mathrm{Ip} \leq \mathrm{To}$; uncertainty area;
$\{\mathrm{Gi}\}$ : set consisted of pixels which have such an intensity Ip that To $<$ Ip $\leq$ Ts; uncertainty area;

$\{G\}$ : set consisted of pixels which have such an intensity Ip that Ip > Ts; almost certainly, these pixels correspond to track points.

Therefore, at the iteration step $\mathbf{t}$ we have:

$$
\begin{aligned}
& S(i, j, t)=J\left(i, j, t, T_{i}\right) \\
& S_{i}(i, j, t)=J\left(i, j, t, T_{i}, T_{0}\right) \\
& G_{i}(i, j, t)=J\left(i, j, t, T_{0}, T_{S}\right) \\
& S(i, j, t)=J(i, j, t, T s)
\end{aligned}
$$

Finally, we have imposed the following congruence conditions between the matrix $\{\mathrm{J}\}$ (cf. Eq. 6) and the previous sets:

some edge pixels calculated by the matrix $\{\mathrm{J}\}$ (cf. Eq. 6) detect closed and connected regions. Depending on pixel intensities and number, each of these regions is considered as a track or as a background. The thresholding sets are then modified in relation to the association;

the edge pixels calculated by the matrix $\{\mathrm{J}\}$ (cf. Eq. 6) which fall into $\{\mathrm{S}\}$ are not useful for determining the track contour (boundaries between background and background); the pixels of $\{\mathrm{Si}\}$ adjacent to $\{\mathrm{S}\}$ can pass from $\{\mathrm{Si}\}$ to $\{\mathrm{S}\}$ if they do not belong to the set $\{\mathrm{J}\}$ (cf. Eq. 6), the same logic can be applied to the sets $\{\mathrm{Gi}\}$ and $\{\mathrm{G}\}$.

A suitable algorithm takes all these aspects into consideration in order to "empty" completely the uncertainty sets $\{\mathrm{Si}\}$ and $\{\mathrm{Gi}\}$, and attributes the pixels initially belonging to them to $\{\mathrm{S}\}$ or $\{\mathrm{G}\}$. Through these observations, the suggested algorithm is able to define the matrix $\mathrm{CF}(\mathrm{i}, \mathrm{j})$ of the final edge, which is formalized by the following relation:

$$
C F(i, j, t)=F\left(S(i, j, t), S_{i}(i, j, t), G_{i}(i, j, t), G(i, j, t)\right)
$$

where $\mathrm{F}$ is the function which is meant to make the abovementioned congruence conditions explicit. Its superimposition on the original image is illustrated in Fig. 11.

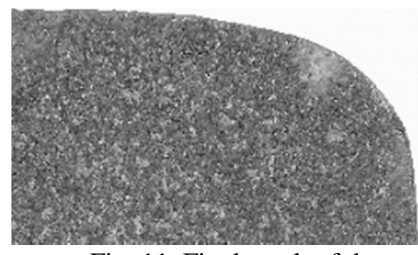

Fig. 11. Final result of the algorithm suggested

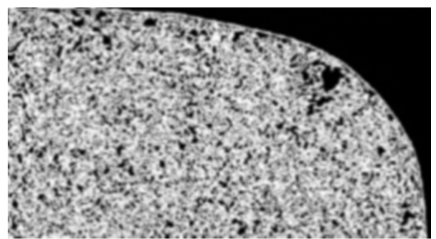

Fig. 12. Binary encoding of the previous track portion
It should be underlined that, downstream from the previous thresholding operations (cf. relation (10)), the procedure is also able to extrapolate not only the exact contour position (cf. relation (11)), but also a first object-based binary encoding which is formalized as follows:

$$
\mathrm{G}(\mathrm{i}, \mathrm{j}, \mathrm{t})=\mathrm{J}\left(\mathrm{i}, \mathrm{j}, \mathrm{t}, \mathrm{T}_{\mathrm{S}}\right)
$$

The result which can be obtained by applying the previous 
relation to the track part in Fig.1, is illustrated in Fig.12. This binary encoding is properly refined on the basis of some binary morphology operations described below. In fact, by carefully examining Figure 12 we note that this binary selection has inside some cavities which do not allow to create a binary large object or blob constituted by close and connected pixels; therefore it requires the application of some mathematical morphology operations. Starting from the previous relation 12 , the procedure modifies the original information by applying some morphological operations and creates the matrix BW illustrated in Figure 14, with regard to the whole track image which clearly shows a complete connectivity between neighboring pixels.

Starting from the Boolean matrix G (cf. relation 12), the algorithm allows to operate on the single bitmaps using a Boolean logic and especially to extract topological and geometric information from a binary image $\mathrm{G} \subseteq \mathrm{E}$ where $\mathrm{E}$ denotes the set of all the possible images of a known dimension. By using a smaller image E, called structuring element - which is a matrix composed of binary values $(0$ or 1) with properly chosen dimensions - it is possible to perform the elementary mathematical morphology operations, i.e. dilation and erosion, also known as Minkowski addition and subtraction [4]. Other operators are also frequently denoted as elementary, more specifically opening and closing transformations achieved by appropriately combining erosion and dilation transformations. In particular, dilation and erosion/Minkowski addition and subtraction are frequently formalized by the two following expressions:

\section{Dilation : $G \oplus B$}

Erosion : $G \Theta B$

where $G$ is the original image matrix and $B$ is the structuring element and $\theta$ and $\theta$ are Minkowski addition and subtraction. These operations are performed as follows:

a. the structuring element is placed in the image so that it is contained in its entirety in the initial figure;

b. the image pixel value, coinciding with the central pixel of the structuring element, results to be equal to that of the structuring element;

c. if the previous condition b) occurs, the structuring element values replace those in the initial image;

d. if the condition b) does not occur, image pixels remain unchanged;

e. the structuring element of one pixel at a time is moved up to cover all the image pixels.

Such operations are generally performed "in a cascade" so as to obtain a final image with precise features. According to their performance order, two different morphological operations are defined:

a. closing: a dilation is performed and followed by an erosion. The closing operation, symbolically expressed with $\mathrm{G}^{\bullet} \mathrm{B}=$ $(\mathrm{G} B \mathrm{~B}$, is useful to eliminate any "hole" and small concavities and to strengthen the connectivity between the regions weakly linked;

b. opening: an erosion is performed and followed by a dilation or $\mathrm{G}^{\circ} \mathrm{B}=(\mathrm{G} B \mathrm{~B}$. Such an operation allows to separate the regions weakly linked and to remove extremely small regions.

It is worthwhile saying that for these operations, the values of the matrix (structuring element) are all set to 1 in the dilation and 0 in erosion. By way of example, Figure 13 illustrates a hypothetical fragment of Boolean image and reports the closing operation results, which clearly show the local discontinuities (holes) to be closed up and the object still dilated at the end of the dilation. The extent of the dilation obviously depends on the structuring element size and shape. At the end of the erosion operation, instead, we obtain an object whose sizes are generally smaller that the original ones $^{2}$. For closing operations, taking into consideration the pixel dimensions of the image and the object of interest (track), a [3x3] structuring element was utilized. The image obtained downstream from a morphological closing operation is illustrated in Figure 14.

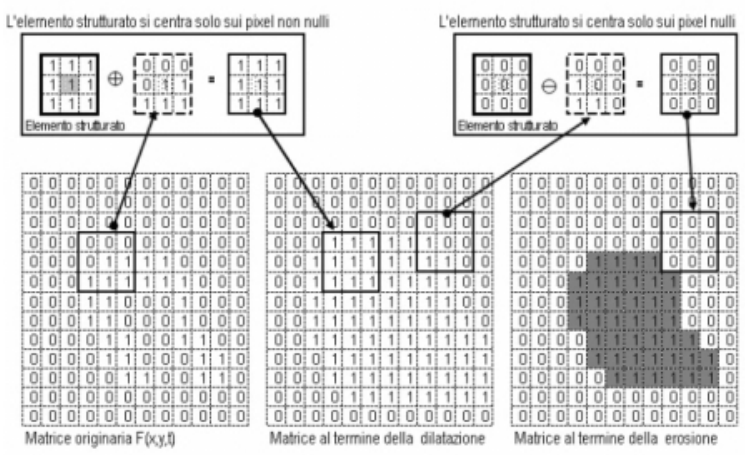

Fig. 13. Dilation, Erosion and Morphological Closing Operations

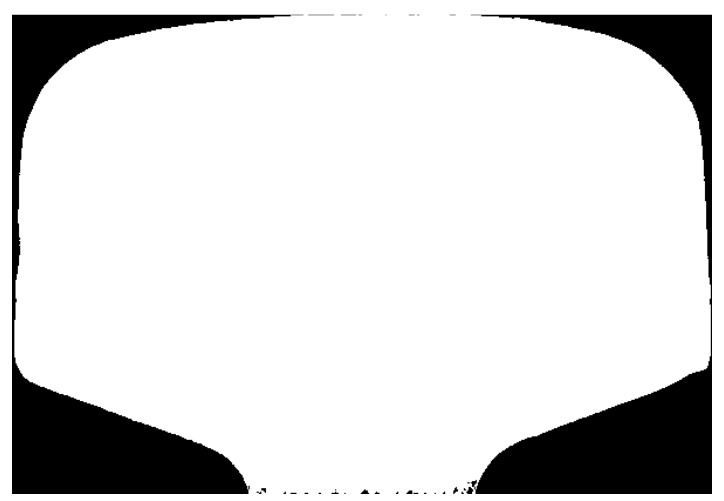

Fig.14. Morphological Closing Operations for the rail under examination

\section{GEOMETRIC REFERENCE-FEATURE EXTRACTION: DEFORMATION DETECTION IN A WORN-OUT RAIL}

For the purpose of this research, we further measured some geometrical sizes which characterize the binary object shape previously defined. Encoding the object edge information can be a compact mode to represent shape and position inside the

${ }^{2}$ Such dimensions are correlated with the Strel structuring element size and shape [3X3, 4X4 etc. matrices] 
image. The most widespread method, commonly known as chaincode, was originally developed by Freeman [5], [6] and consists in storing a list of points (without any color but only position information). The basic idea is to run along the object contour and to encode progressively the direction to follow. Once determined the coordinates of a point in the object border (generally at the highest and the leftmost position), the successive point is detected only by following the direction which connects pixel barycenters [7].

It is here worth remembering that, by convention, the coordinates are always referred to a Cartesian orthogonal system which originates in the first pixel at the top left side of the image. Obviously, in digital image analysis, the exact origin position is set so as to associate every pixel (square element in the image matrix) to Cartesian coordinate planes (xi, yi), which, therefore, allow to detect metrically the pixels in the digital image. In order to do that, the following convention is usually adopted:

- $\mathrm{x}$-axis parallel to the upper side of the digital image and oriented towards right;

- $y$-axis parallel to the left side of the digital image and oriented downwards;

- origin shifted by half a pixel to the upper left side with regard to the top left-hand corner of the digital image.

In such a system, the pixel can be set equal to a finite dimension of $\Delta \mathrm{x}$ and $\Delta \mathrm{y}$ unities, so as to determine a objective mapping between the pixel position ( $\mathrm{i}=$ row, $\mathrm{j}=$ column, with matrix reference) and the Cartesian coordinate plane system (xi,yi).

In fact the pixel barycenter (or square center) appears to be detected as indicated in the following explanatory figure which denotes $\Delta \mathrm{xi}$ barycenter $=\mathrm{i} \bullet \Delta \mathrm{xi}$ and $\Delta \mathrm{yi}$ barycenter $=$ $\mathrm{j} \cdot \bullet \mathrm{yi}$

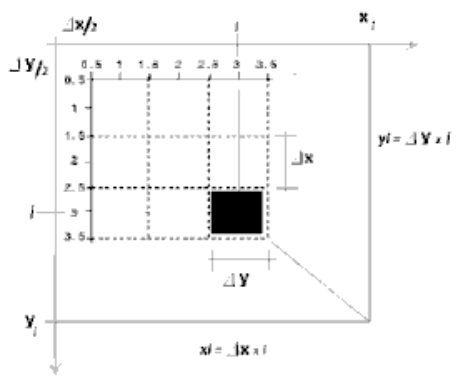

Fig. 15. Pixel barycenter detection

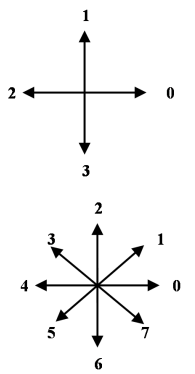

Fig.16, Fig. 17 -Connectivity Neighbors Figure
All this considered, the directions admitted are usually restricted in order to increase the coding efficiency.

Figures 16 and 17 show some of the most frequently adopted conventions:

1. in case of 4-connectivity it is possible to proceed only horizontally or vertically;

2 . in case of 8-connectivity it is possible to proceed both diagonally and horizontally and vertically;

3. in case of 6-connectivity the deviations are marked following the pixel edge (not between the barycenters as happens, instead, in the two previous cases).

Though more natural, the latter encoding requires the recourse to more complex and less performing algorithms. The directions to follow are conventionally identified as integers (4 or 8 paths). By applying an "edge descriptor", derived from an 8-path algorithm, to the object schematically illustrated in Figure 18, the following encoding is obtained: [0701766344342].

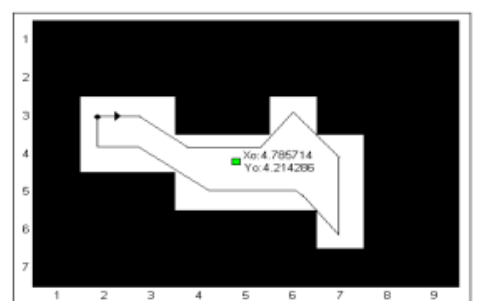

Fig. 18. Chaincode algorithm application to a binary object

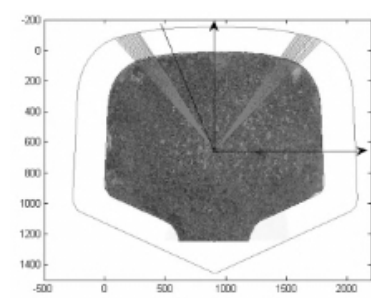

Fig. 19. Detection of the rail head auxiliary edge
Once detected the blob (binary large object) and its contour coordinates, it is simple to calculate the following geometric object data [3], [6]:

1. area (A): expressed in terms of pixel number representing the object;

2. perimeter $(\mathrm{P})$ : pixel number forming the contour denoted with P;

3. compactness (C): given from the following relation $\mathrm{C}=\mathrm{P} 2 / \mathrm{A}$ (the square of the perimeter makes the relation independent of the real dimensions of the object).

4. centroid (M) representing the center of the masses (center of gravity)

More specifically, area and perimeter are sizes dependent of geometric transformation operations carried out on the image, whereas compactness is a topological parameter of the object and expresses the smaller or greater complexity of the edge (more or less indented). The centroid is the geometrical center of the blob; it expresses the mean position of the white pixels in the Boolean image and is in fact defined by calculating the mean between the axes $\mathrm{x}$ and the ordinates $\mathrm{y}$ with regard to all the white pixels which are contained in the same Boolean image. Referring to the image in Figure 18, this procedure is formalized as follows:

$$
\begin{aligned}
& \text { Mean } \mathrm{x}=\frac{1}{\text { axis number }} \sum_{\mathrm{k}=1}^{\text {white pixels }} \mathrm{i} * \mathrm{~B}(\mathrm{i}, \mathrm{j}) \\
& =\left(\frac{1}{14}(2+2+3+3+4+4+5+5+6+6+7+7)\right)=4.785
\end{aligned}
$$

Mean $\mathrm{y}=\frac{1}{\text { ordinate number }} \sum_{\mathrm{k}=1}^{\text {white pixels }} \mathrm{i} * \mathrm{~B}(\mathrm{i}, \mathrm{j})$

$=\left(\frac{1}{14}(3+4+3+4+4+5+4+5+3+4+5+4+5+6)\right)=4.211$

We then determined the centroid position (xoj, yoj) of the worn-out track and its contour coordinates, got from Freeman's algorithm developed on the result formalized by relations (11) and (12) after carrying out the proper morphological closing operations (cf. Fig. 14). The contour 
coordinates are collected by vector WT (worn-out track) of dimension [ $\mathrm{n} \times 2$ ], where $\mathrm{n}$ is the Cartesian axis number of the edge. The algorithm then adds the profile coordinates of the unworn track (in this case UIC 60 tracks have been taken into consideration) and carries them to the same image scale previously determined. These coordinates are properly recorded in a vector IT (intact track) of dimension [kx2] where $\mathrm{k}$ represents the number of the discrete axes denoting the contour of the intact track.

In order to get the deviation between the profiles of the worn-out and the unworn tracks, an auxiliary profile has been developed by reducing the previous IT by a constant term equal to $1.3(\mathrm{Rg})$, thus obtaining a perfectly homothetic boundary to the profile with the coordinates in the vector IT. The following Figure 19 is shown to this end.

The distances between the two profiles have been determined by the intercepts of the straight lines from the centroid of the profile IT, with regard to this last boundary $\mathrm{Rg}$, whose domain is obviously perfectly parallel to the previous one. The procedure allows to get the deviation between the two compared profiles (worn-out and unworn) with regard to a $360^{\circ}$ rotation of the straight line coming out of the track centroid and, therefore, produces output information on the wear condition of the whole track profile. Figure 20 shows the final result yielded by the application of the algorithm suggested. By way of example, the following diagram illustrates the trend of the deformation of a UIC60 rail profile properly examined in laboratory to validate the effectiveness of the method suggested, with regard to the part of the rail head located at an angle " $\theta$ " between $20^{\circ}$ and $160^{\circ}$. By doing so, it is also possible to work out the values of vertical, horizontal and $45^{\circ}$ wear, as required by the technical rules approved by the Italian Railway Network [8], [9].

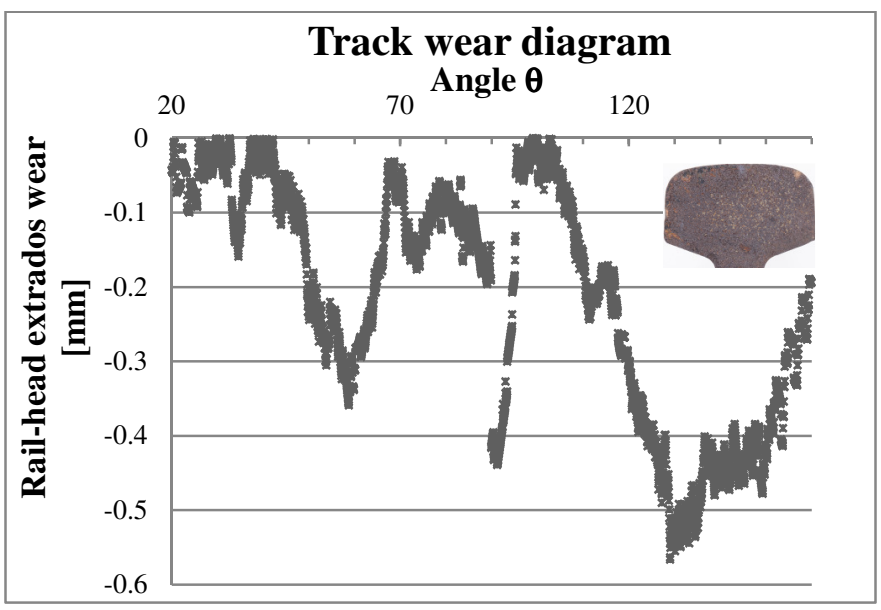

Fig. 20. Point wear of the rail-head

\section{CONCLUSIONS}

Monitoring the wear condition of the railway superstructure is one of the key points to guarantee an adequate safety level of the railway transport system. With regard to such a field, in this paper a new non-conventional procedure has been examined to determine the track wear, based on the image processing technique.

The method, based on the analyses of high-resolution photographic images, has required the working out and the following implementation of specific mathematical algorithms able to provide the cross section geometry of a generic wornout track and the measurement of the relevant deviations compared to new tracks of the same typology.

At present, the procedure can be only used for the lab analyses showing high precision in the wear evaluation as well as great rapidity in being performed.

In light of the best results yielded, further (stereoscopic) methodologies are being developed to reconstruct the track profile through equipment installed directly on diagnostic carriages. So, it is very likely that a laser monitoring system will be combined with a digital one in the future.

\section{REFERENCES}

[1] J. Canny, "A computational approach to edge detection", PAMI(8), No. 6, November 1986, pp. 679-698

[2] R.C. Gonzales, R. E. Woods "Digital Image Processing 2nd ed." Prentice Hall, Upper Saddle River, New Jersey, (2002)

[3] R.C. Gonzales, R.E. Woods, S.L. Eddins, "Digital image processing using Matlab", Prentice Hall, Upper Saddle River, New Jersey, 2004

[4] Serra J., "Image analysis and mathematical morphology", London, Academic Press, 1982

[5] Freeman, H., "Computer Processing of Line Drawing Images" Computing Surveys. 6(1):57-97

[6] H. Freeman, "On the encoding of arbitrary geometric configurations", Ire Trans. Electron. Comput. EC-10, 260-268, 1961

[7] X. Trouillot, M. Jourlin and J.C. Pinoli, "Geometric parameters computation with freeman code", submitted to Image anal. Stereol., 6 pages

[8] Procedura operativa subdirezionale RFI DMAIMSD P0 IFS 0020 del 16.01.02, Rilievi della geometria del binario e relative disposizioni manutentive, Rete Ferroviaria Italiana, 3.2002

[9] D. Di Vincenzo, M. Guerrieri, "Gestione e diagnostica della sovrastruttura ferroviaria. un caso studio: la linea Palermo - Messina", Argomenti ISSN 1973-4220- Rivista quadrimestrale di RFI dicembre 2005, N. 7, pp. 47-77. Available: http://www.trenitalia.com/cmsfile/allegati/rfi/ARGMENTI_7.pdf 


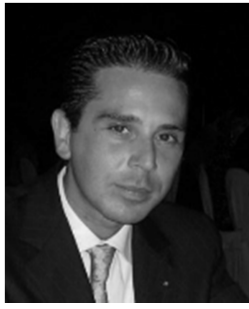

Marco Guerrieri, born in Palermo on 24/12/1976, has been graduated in 2001 cum laude in Civil Transportation Engineering (degree course lasting five years). He received the $\mathrm{PhD}$ in Road Infrastructure Engineering from the Faculty of Engineering, University of Palermo (Italy), in 2006. He was Postdoctoral researcher at the Department of Road Infrastructure Engineering from 2006 to 2009. Since August 2011, he is Postdoctoral Researcher at CRB (Faculty of Engineering), Perugia University. Designed as Expert of the ICAR04 disciplinary sector (Road, Railway and Airport) at the University of Palermo from 2006 to 2009 and since May 2011 at the University of Enna "Kore". From 2006 to 2011 he was professor in several university masters courses and, in the academic year 2010/2011, professor of Transport and mobility infrastructures (Faculty of Architecture, University of Palermo).

Eng. Guerrieri is member of Italian Society of Transport Infrastructures (SIIV), Italian Railway Engineers College (CIFI), Italian Association of Traffic and Transport Engineering (AIIT). He is author of over 35 papers, patent of inventions and technical notes published on national and international congress and journals.

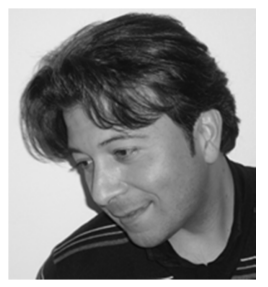

Giuseppe Parla, born in Caltanissetta on 27/6/1973, has been graduated in 2003 cum laude in Civil Transportation Engineering (degree course lasting five years). He received the $\mathrm{PhD}$ in Road Infrastructure Engineering from the Faculty of Engineering, University of Palermo (Italy), in 2008. Since 2008, he is Postdoctoral researcher at the Department of Road Infrastructure Engineering, University of Palermo. During 2008/2009, 2009/2010 and 2010/2011 academic years he also collaborated on teaching activities of "Road Infrastructures" course of Civil Engineering, University of Palermo. From 2006 to 2011 he was professor in several university masters courses.

Eng. Parla is member of Italian Society of Transport Infrastructures (SIIV), and author of numerous scientific publications.

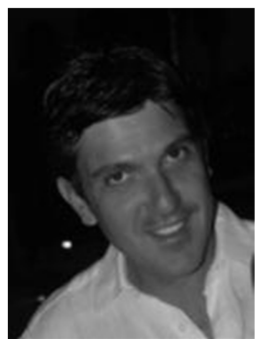

Dario Ticali, born in Palermo on 6/12/1975, has been graduated in 2001 in Civil Transportation Engineering (degree course lasting five years). $\mathrm{He}$ received a master's degree in Project Management and Public Policies (academic year 2001/2002) and $\mathrm{PhD}$ in Road Infrastructure Engineering from the Faculty of Engineering, University of Palermo, in 2006. Since 2008, he is Researcher and professor of Transport engineering infrastructures at Faculty of Engineering and Architecture of "Kore", University of Enna (Italy). He is Chair of IPPC (Integrated Prevention and Pollution Control) Committee, nominated by Environment Ministry of Italian Government (Since 2008), component of National Committee for Industrial Research Scientific Project, nominated by Research and University Ministry of Italian Government (since 2009) and Chair of Regional Committee for Environmental Protection, Reclamation and Water Reuse of Sicily Region, nominated by Chair of Ministry Council of Italian Government (since 2010).

Eng. Ticali is member of Italian Society of Transport Infrastructures (SIIV), and author of over 25 papers, published on national and international journals and/or presented at scientific congresses. 